\title{
Novel techniques for increasing the size of the radial artery during ultrasound-guided cannulation
}

\author{
Praveen Maheshwari, MD • Bradley Kelsheimer, MD • Parul Maheshwari, MD
}

Received: 13 October 2015/Revised: 22 October 2015/Accepted: 13 November 2015/Published online: 20 November 2015

(C) Canadian Anesthesiologists' Society 2015

\section{To the Editor,}

With more widespread availability of portable ultrasound (US) equipment, there has been an increase in the use of US for arterial and venous cannulation. Though challenged by some, ${ }^{1}$ the use of US guidance for arterial cannulation has been reported to increase the rate of success, reduce the time required for placement, and decrease the rate of complications. $^{2}$

Despite the potential benefits of US to guide radial artery cannulation, it is often reserved only for difficult situations when palpation or Doppler methods are unsuccessful. In certain circumstances, even US guidance may prove to be difficult secondary to hypotension, hypovolemia, vasospasm, peripheral vascular disease, as well as user inexperience. The initial assessment of the site for radial arterial cannulation is usually performed by placing an US probe perpendicular to the direction of the artery about $2-3 \mathrm{~cm}$ proximal to the distal wrist crease (Figure A). This should reveal a short-axis view of the radial artery (Figure B). Identification of the artery can be confirmed by gently increasing the pressure of the US probe over the artery and evaluating the compressibility of the visualized structures-i.e., the relatively noncompressible artery can be identified often flanked by highly compressible radial veins. Additionally, longitudinal imaging utilizing colour flow Doppler can ascertain the pulsatility and direction of blood flow. Furthermore, if needed, the US probe can be moved more proximally along

P. Maheshwari, MD $(\varangle) \cdot$ B. Kelsheimer, MD .

P. Maheshwari, MD

Department of Anesthesiology, The University of Oklahoma

Health Sciences Center, Oklahoma City, OK, USA

e-mail: Praveen-Maheshwari@ouhsc.edu the radial artery to identify a more optimal site for cannulation.

Herein, we report several novel techniques to increase radial artery diameter by amplifying the characteristics of blood flow through the artery. This may be helpful during situations when conventional methods for cannulation have proven unsuccessful.

The first of the techniques involves holding the US probe between the index and middle finger and placing it over the radial artery. By utilizing the index finger to apply pressure, the radial artery is occluded distal to the probe leaving space to perform arterial cannulation (Figure C). A short-axis US view of the radial artery is obtained, and an increase in the size of the lumen can be observed due to the distal outflow obstruction (i.e., from the digital pressure). This leads to an increase in back pressure and subsequent enlargement of the diameter and cross-sectional area of the radial artery (Figure D). Theoretically, the increase in the size of the artery when utilizing this technique may improve the cannulation success rate, reduce the time to cannulation, and possibly even lessen the complications of radial arterial cannulation due to a reduction in the number of cannulation attempts.

The second proposed technique involves compression of the ulnar artery (by an assistant) at approximately 1-2 cm proximal to the distal wrist crease (Figure E). The occlusion of the ulnar artery may improve the blood flow through the radial artery and, as a result, increase the radial artery diameter and area (Figure F). The contribution of the occlusion of the ulnar artery to improve the radial arterial blood flow may be less than expected in patients with a positive Allen's test. ${ }^{3}$

Theoretically, combining the above two techniques could further enhance the radial artery diameter. Future study might elaborate on the usefulness of these techniques to improve cannulation success rates. 


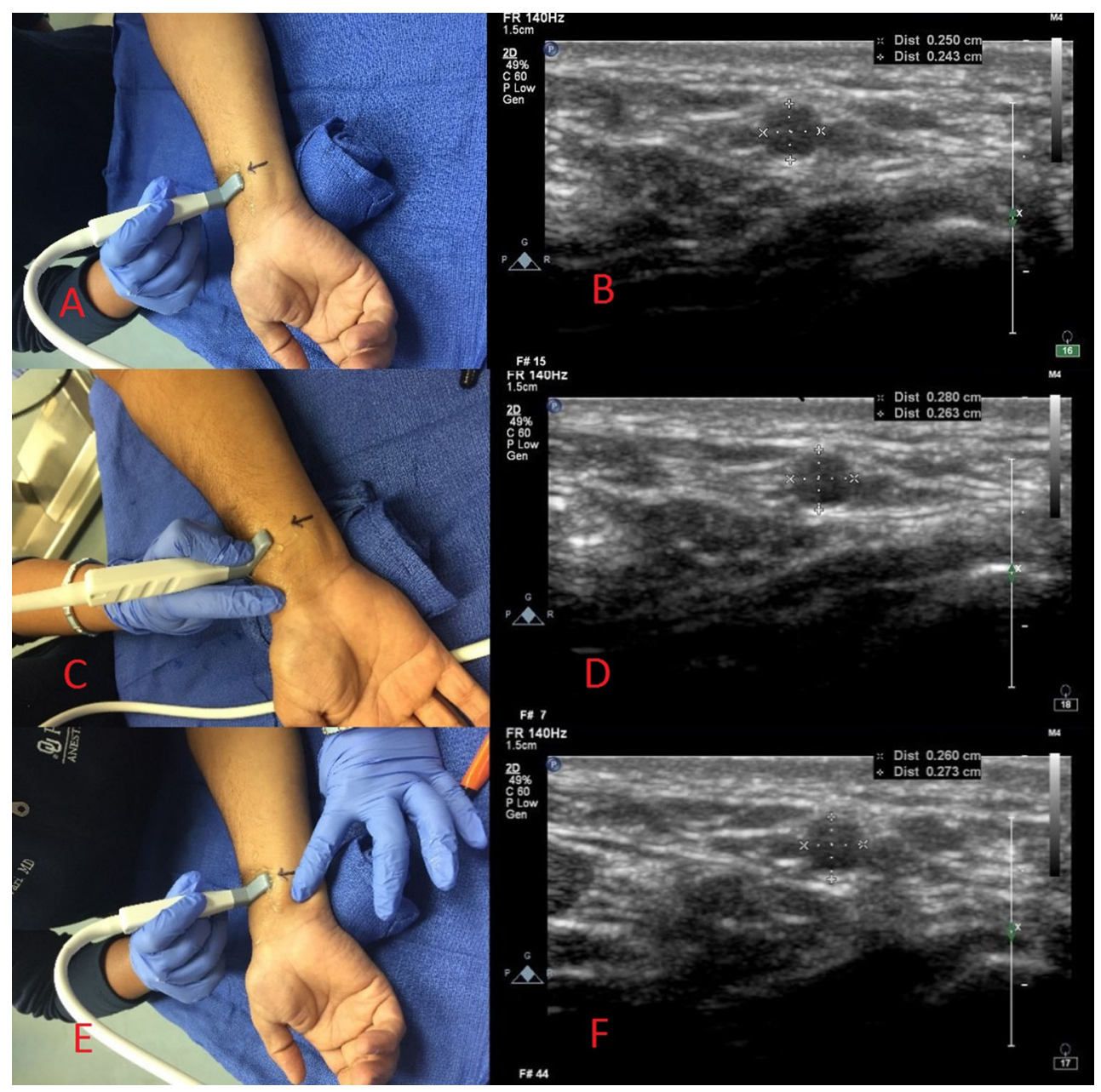

FIGURE The arrows in the above figure indicate the level at which the ultrasound (US) image is obtained and further illustrate that all images were obtained at the same level. Additionally, these images are strictly to demonstrate the novel techniques, and a sterile technique is encouraged during radial artery cannulation with US. An US probe is placed over the radial artery in a perpendicular position approximately $2-3 \mathrm{~cm}$ proximal to the distal wrist crease (Figure A) to obtain a short-axis US image (Figure B). As the US

Acknowledgement Kate Strum (same affiliation as authors) assisted in the writing, editing, and preparation of this document.

Conflicts of interest None declared.

Funding None.

\section{References}

1. Peters C, Schwarz SK, Yarnold CH, Kojic K, Kojic S, Head SJ. Ultrasound guidance versus direct palpation for radial artery probe is held between the index and middle finger (Figure C), pressure is applied with the index finger to occlude the distal radial artery. This increases the diameter and hence the cross-sectional area of the artery (Figure D). Figure $\mathrm{E}$ shows that additional digital pressure can be applied by an assistant to occlude the ulnar artery, with the corresponding short-axis US image (Figure F) showing the increased size of the radial artery

catheterization by expert operators: a randomized trial among Canadian cardiac anesthesiologists. Can J Anesth 2015; 62: 11618.

2. Gu WJ, Wu XD, Wang $F, M a Z L, G u X P$. Ultrasound guidance facilitates radial artery catheterization: a meta-analysis with trial sequential analysis of randomized controlled trials. Chest 2015; DOI: $10.1378 /$ chest.15-1784.

3. Tanzilli $G$, Truscelli $G$, Barilla $F$, et al. Evaluation of hand circulation with CardioWaves photoplethysmograph device during Allen test in healthy volunteers. Eur Rev Med Pharmacol Sci 2015; 19: 3006-11. 